\title{
Toddlers can use semantic cues to learn difficult nonadjacent dependencies
}

\author{
Jon A. Willits ${ }^{*}$, Jill Lany ${ }^{2}$, Jenny R. Saffran ${ }^{3}$
}

\author{
1. Department of Psychology \\ University of California, Riverside \\ 900 University Avenue \\ Riverside, CA 92521 \\ 2. Department of Psychology \\ University of Notre Dame \\ Haggar Hall, Room 118-C \\ Notre Dame, IN 46566 \\ 3. Department of Psychology \\ University of Wisconsin \\ 1202 W. Johnson Street \\ Madison, WI 53706 \\ * Corresponding author
}

\begin{abstract}
Nonadjacent structure (where one element predicts another, but at a distance) is a hallmark of natural languages. The fact that infants and adults have difficulty learning nonadjacent structure in laboratory settings has led to theories suggesting that there are strict constraints on the units over which humans track nonadjacent structure. In four experiments, we tested the hypothesis that correlated semantic cues enhance toddlers' ability to learn nonadjacent linguistic structures. Toddlers successfully transferred these patterns to new strings spanning novel intervening items, and to novel nonadjacent pairs drawn from the same semantic categories. The results provide evidence that toddlers can use rich, redundant input that includes meaning to learn nonadjacent structures akin to those present in natural languages.
\end{abstract}

Keywords: toddlers; language acquisition; statistical learning; syntax; semantics 


\section{Introduction}

Natural languages contain complex patterns and regularities at many levels, giving rise to an expressive power unmatched by any other communicative system. Some of these patterns can be characterized by local predictive relationships, such as the co-occurrence probabilities amongst phonemes, syllables, and words. Across development, humans are exquisitely sensitive to these adjacent regularities, suggesting that this form of learning plays an important role in language acquisition (1).

However, natural languages also contain structure that transcends simple associations between adjacent elements. Nonadjacent dependencies - in which one unit predicts a second unit, but at a distance - exist in all human languages, and at virtually every level of linguistic structure. There are nonadjacent dependencies (also sometimes called long-distance dependencies) between low-level units like phonemes. For example, nonadjacent sequences of consonants form word roots in many languages (e.g., English irregular verbs like $\underline{\operatorname{drink}} \underline{\underline{d r}} \underline{\underline{n} k}, \underline{d r} \underline{\underline{u n k}}$; noun frames in Semitic languages). There are also nonadjacent dependencies between higher-level units, such as number agreement between main noun phrases and verb phrases in English (e.g., the cat sleeps vs. the cats sleep), which can be separated by embedded clauses (e.g., the cats that I saw yesterday sleep in the shed). Nonadjacent dependencies in grammatical structure convey critical information (). They vastly increase the complexity of language and the information that it contains. They also vastly increase the complexity of language learning.

Adjacent and nonadjacent patterns are not limited to dependencies between specific units, but also extend to relations between the abstract classes to which these units belong. Chomsky's (3) classic "colorless green ideas sleep furiously" example demonstrates a relationship between abstract grammatical classes, not just between specific words. It has been argued that many of the important adjacent and nonadjacent relations exist as relations between abstract representations (such as semantic and grammatical classes), rather than as relations between specific words or morphemes (4). However, research has provided evidence for a more pluralistic view, with linguistic representations being composed of a combination of abstract and item-specific relations (5-9).

Given the importance and ubiquity of nonadjacent dependencies in language (as well as in other domains of cognition), it is not surprising that children show early sensitivity to these structures in their native language. For example, 18-month-old infants discriminate between grammatical and ungrammatical sentences differentiated by the grammaticality of the match between a verb's auxiliary and inflection (e.g., is cooking versus *can cooking) (10). Likewise, in sentences like "Chris has a red ball but Max doesn't have one," 17-month-olds expect the anaphor one to refer to the pragmatically-implied red ball, not simply to ball (11). By 3 to 5 years of age, children can correctly link pronouns to nouns within complex sentences and larger units of discourse (12). More generally, children acquire nonadjacent dependencies involving complex semantic, pragmatic, and discourse structures. This includes relationships between words in alternative syntactic structures (e.g., active sentences versus passive sentences), different word meanings (13-14), and the use of prior contexts to correctly comprehend ambiguous input, even when these prior contexts do not immediately precede the ambiguous element (1517).

Adults are sensitive to an even larger range of nonadjacent dependencies involving phonology, morphology, syntax, and semantics (18). Furthermore, experienced language users are sensitive to both the abstract and item-specific dimensions that relate nonadjacent elements. For example, adults possess general knowledge about verbs' argument structures, such as the number and types of nouns that are required by each verb, and that subject nouns and verbs must match in number. Adults also have expectations about the arguments that typically co-occur with specific verbs, such as that the verb give is likely to have an animate agent as its subject and an inanimate direct object (19-20), or that pasta takes a singular verb while noodles takes a plural verb (21-22).

Given these facts about the nonadjacent structures present in natural languages and the manner in which they are mastered, there is an intriguing disconnect that merits explication. On the one hand, nonadjacent dependencies are ubiquitous in natural languages, and people clearly learn them during the course of everyday language acquisition. However, experimental paradigms have found that adults have 
considerable difficulty learning new nonadjacent dependencies when there is not some indirect cue to the nonadjacent relationship. Learners from infants to adults successfully learn novel nonadjacent dependencies in a range of lab tasks. But when the nonadjacent dependency is the only thing to be learned, and when there is no external or correlated cue to that nonadjacent dependency, learners often fail to acquire it. Some other cue or bootstrapping mechanism often seems to be necessary to facilitate this form of learning.

The paradigm typically used to study nonadjacent dependency learning involves familiarization with strings of nonsense phonemes, syllables, or words that contain nonadjacent dependencies. In a classic study, Gómez (2002) familiarized 18-month-olds with word strings drawn from an AxB grammar, where a particular A-word perfectly predicted which B-word would occur after an intervening x-word (23). Following familiarization, the infants were tested on their ability to discriminate familiarized strings containing the nonadjacent dependency from novel strings that violated the dependency. Gómez found that both infants and adults failed to learn this deceptively simple structure. More broadly, the literature contains a mixture of studies that contrast successful learning of nonadjacent dependencies with studies that show failures to learn. What factors predict success versus failure?

One important distinction appears to be the grain size over which the structure is organized. Most of the studies that show successful learning involved nonadjacent dependencies between units of relatively small grain size, such as consonants, vowels, or syllables (24-31). For example, Peña et al. (1999) trained adult participants on sequences containing syllable triplets where the first element predicted the third element with variability in the middle element (e.g. "PU-RA-KI, BE-LI-GA, PU-FO$\underline{\mathrm{KI}}, \mathrm{BE}-\mathrm{RA}-\mathrm{GA}$ "). They found that participants learned the nonadjacent structures when the triplets were bracketed with short pauses, but not when the sequence consisted of an unbroken stream of syllables.

Newport and Aslin (2004) contrasted two different types of non-adjacent patterns: between sequences of $\mathrm{CV}$ syllables (e.g. BA-x-TE, GU-x-DO) versus between consonants ( $\underline{\mathrm{Pa}} \underline{\mathrm{G} o} \underline{\mathrm{Te}}$, $\underline{\mathrm{Pae}} \underline{\mathrm{G}} \underline{\mathrm{T}} \mathrm{u}$, $\underline{\mathrm{D}} \underline{\mathrm{K}} \mathrm{B} \underline{\mathrm{Be}}, \underline{\mathrm{D}} \mathrm{a} \underline{\mathrm{K}} \underline{\mathrm{Bu}}$ ) (27). The results revealed that while participants could learn the consonant-based non-adjacencies, they failed to discover the syllable-based non-adjacencies. Newport and Aslin suggested this may be due to a perceptual anchoring effect; the nonadjacent consonants all shared a number of perceptual features in common, which effectively created two streams of data in which statistics could be tracked separately (leading to an adjacent dependency within the consonant stream). Newport and Aslin argue that the sequences of CV syllables have no such anchoring cue, and thus nonadjacent dependencies within the stream are harder to learn. Creel, Newport, and Aslin (2004) (32) provided additional support for this account by showing that nonadjacent tone sequences that share perceptual features are also easier to learn (though see Bonatti et al., 2005, for an alternative explanation) (28).

Research at the lexical level supports the interpretation that learners find nonadjacent dependency learning to be difficult, when there is no external cue to the dependency. As noted, when Gómez (2002) presented infants with strings of multisyllabic words following a simple 2x3 AxB grammar (two nonadjacent $\mathrm{AB}$ pairs with three intervening items for a total of six strings), infants did not learn the dependency (23). However, learning did occur when the set size of intervening items was relatively large (12-24 items), creating a distributional situation with more variable adjacent dependency statistics. This distributional landscape likely called attention to the nonadjacent relations, which were more informative than the adjacent statistics (33-35). Related research suggests that both adults and infants are better able to learn non-adjacent dependencies when they had previously experienced them as adjacent dependencies (36-37). As in the studies involving phoneme-level dependencies, nonadjacent dependencies between words appear to be relatively hard to learn in lab tasks, unless something (in this case about the structure of the input, past or present) called attention to the dependency.

One of the most intriguing issues concerning nonadjacent dependency learning is the role of correlated cues. Bonatti, Endress and colleagues $(23-32,38)$ argue convincingly that this issue is complex, and ambiguous with regard to nonadjacent dependencies at the phonemic level (such as in the findings from Newport and Aslin, 2004). However, the effect seems to be quite robust at the lexical level. Van den Bos, Christiansen, and Misyak (2012) found that adults more easily learned a probabilistic nonadjacent 
dependency if the items shared phonological features, or if the auditory string was paired with a visual stimulus such that the nonadjacently related items were also visually more similar to one another (39). Indeed, Christiansen (2013) has argued that language has evolved to depend on these multiple cue integration processes (40). Endress and Hauser (2009) conducted a study demonstrating further evidence that some (but not all) correlated cues help learn complex structure (41). They trained adults on sequences of three-word strings that followed an $\mathrm{ABB}$ and $\mathrm{AAB}$ pattern. However, unlike previous studies using this paradigm (42-44), the first and third items were not the exact same item, but rather were different items drawn from the same semantic categories (animals or foods). Endress and Hauser found that adults learned the $\mathrm{AAB} / \mathrm{ABB}$ rule when the items were drawn from the same class.

These last two examples (Endress \& Hauser, 2009; Van den Bos et al., 2012) are particularly intriguing because of their implications for nonadjacent dependencies correlated with meaning. Many higher-level nonadjacent dependencies in natural languages (such as those between verbs and their noun arguments) have semantic correlates. For example, it is not an accident that ditransitive verbs like give typically co-occur with three nouns (an agent, a patient, and a recipient); the meaning of give requires these three relations. Likewise, the types of nouns that typically fill these roles are not random or unpredictable: a verb like give (at least in its literal meaning) must have an animate agent that does the giving. Thus, a language learner acquiring nonadjacent structural dependencies between words in sentences (such as between a subject of a ditransitive sentence and its verb) may be assisted by the fact that there is a meaningful relationship correlated with the structure they need to learn. Learners may be sensitive to these correlations between semantic or conceptual relations and linguistic structure. On this view, learners may use their knowledge about semantic relations, when available, to bootstrap learning about nonadjacent linguistic structure. Indeed, the presence of semantic information plays a critical role in adult processing of combinatorial dependencies in natural and artificial languages (45-47).

The following experiments were designed to test the hypothesis that correlated semantic cues facilitate non-adjacent dependency learning in young language learners. There are a number of ways in which word pairs can be "semantically similar", and some types of similarity may serve as better learning cues than others. Words may be members of the same taxonomic category (48), and share overlapping semantic features (49-50). Words' referents may jointly participate in specific events (51-52). Words may co-occur frequently (53-54). Finally, words may jointly and consistently participate in specific thematic relations (55), linguistic constructions (56), or linguistic contexts (57-58). In order to maximize the likelihood of finding positive evidence to suggest that toddlers can exploit semantic cues correlated with nonadjacent linguistic structure, we chose items that maximized similarity along as many of these dimensions as possible.

We familiarized 24-month-olds with an AxB grammar, similar to the small set-size condition from Gómez (2002) and other studies where children and adults typically fail to learn nonadjacent relationships. However, instead of using nonsense words, we used familiar English nouns for the nonadjacent A and B elements (dog, kitty, cookie, banana) and as the intervening x elements (towel, soap, toothbrush). We chose these nouns because they are known by most two-year-olds according to parental report (59). Using words with known semantic properties allows us to test the hypothesis that toddlers can learn nonadjacent pairs when a semantic cue (here, taxonomic category: cookie-x-banana; dog-x-kitty) is correlated with the nonadjacent structure. Crucially, we compared learning nonadjacent dependencies with correlated semantic cues (Semantic Match condition) against a control condition in which the same words were reordered so that semantic information was not correlated with the nonadjacent structure (Semantic Mismatch condition: cookie-x-kitty; dog-x-banana). Crucially, all test items were auditoryonly, with no referents present, to remove potential confounds of visual similarity during testing. We hypothesized that the presence of correlated semantic cues to nonadjacent structure in the Semantic Match condition would facilitate the acquisition of nonadjacent dependencies relative to the Semantic Mismatch control condition. 


\section{Experiment 1}

The goal of Experiment 1 was to determine whether nonadjacent dependency learning is facilitated by correlated semantic cues. Toddlers were familiarized with an audio-visual corpus following an $\mathrm{AxB}$ grammar, and then tested on auditory-only sequences that either followed or violated the grammar. In each three-word string, the first word (A) perfectly predicted the occurrence of the third word (B), while a set of three intervening words (x) occurred unsystematically across sentences containing the two dependencies. We chose a set size of three x-words because Gómez (2002) found that toddlers were unable to learn a nonadjacent dependency given a small set of intervening elements. To test the hypothesis that semantic cues facilitate learning of nonadjacent linguistic structure, toddlers were randomly assigned to two familiarization conditions. In the Semantic Match condition, the words forming the nonadjacent dependencies were drawn from the same taxonomic category: cookie-x-banana and doggy-x-kitty. In the Semantic Mismatch Control condition, the words were rearranged so that the nonadjacent dependencies linked words from different categories (cookie-x-kitty and doggy-x-banana). This condition was designed to control for other cues to the nonadjacent dependency. These materials may be easier to track and remember than those used in previous studies with the same grammar (e.g., Gómez, 2002), due to the use of audio-visual stimuli during training, stimuli consisting of familiar meaningful words, etc. If so, then toddlers should learn in both the Semantic Match and the Control conditions. Likewise, if toddlers are able to learn regardless of semantic relationships because the key words were placed in highly salient positions like string beginning and ending (as suggested by Endress et al., 2005), then they should learn in both conditions. However, if a correlated semantic cue is instrumental (or at least extremely helpful) in learning the dependency, then the toddlers may show learning in the Semantic Match condition but not the Control condition.

There are many types of semantic relationships that could bind words together across nonadjacent structures. We chose to manipulate taxonomic category because there is evidence that 24-month-old children are sensitive to the similarity of words that belong to these categories $(60,61)$. Of course, these items could also be considered similar along a number of other dimensions, including semantic feature overlap, distributional similarity, and joint participation in linguistic and nonlinguistic contexts. To highlight the semantic cues, pictures of referents were paired with the spoken words during familiarization; the pictures were not presented during the test phase. To ensure that our participants knew the meanings of the nouns, the toddlers (24-month-olds) were slightly older than the participants in prior research on nonadjacent dependency learning.

All participants received the same set of test items: half were sequences presented during familiarization (Familiar test trials) and the other half violated the familiarized nonadjacent structure (Novel test trials). Items that were familiar for the toddlers in the Semantic Match condition were novel for toddlers in the Control condition, and vice versa. Successful discrimination between Familiarized and Novel trials would constitute evidence of non-adjacent dependency learning.

\subsection{Method}

\subsubsection{Participants}

Forty-eight toddlers (24 females) from English-speaking families participated in the study (mean age 24.4 months; range: 22.1 to 25.9 months), and were randomly assigned to one of the two different familiarization conditions (Semantic Match condition: 12 females, mean age 24.2 months; Control condition: 12 females, mean age 24.6 months). All of the participants were typically developing and fullterm according to parental report. Five additional toddlers were tested but did not complete the experiment due to fussiness.

\subsubsection{Materials}

There were two between-subjects familiarization conditions: Semantic Match (the words comprising the nonadjacent dependency were drawn from the same taxonomic category) and Control (the 
words comprising the nonadjacent dependency were drawn from different taxonomic categories). Each familiarization corpus consisted of six sequences, shown in Appendix 1. Each sequence was presented four times for a total of 24 sequences $(2 \mathrm{~min}$ ). The sequence order was pseudo-randomized such that the transition probability between words at sequence boundaries was 0.5 (i.e., sequences ending in banana and kitty were both followed by sequences starting with cookie half the time, and by sequences starting with $d o g$ half the time). As a consequence, the only reliable word-word contingencies in the study lay between the first and third word in each sequence.

All toddlers heard the same 8 test items, shown in Table 1, consisting of 4 familiarization sentences from each condition. For each participant, 4 of these items were Familiar and 4 were Novel, depending on familiarization condition. Because the adjacent transitions in the consistent and inconsistent strings occurred equally often during familiarization in both conditions, this information could not be used to discriminate between test sequences.

The familiarization corpora and test items were constructed to be as similar as possible to the materials used by Gómez (2002). An adult female using a child-directed speech register was recorded speaking each of the seven words (dog, kitty, cookie, banana, soap, towel, toothbrush) in citation form. The isolated words were concatenated with $250 \mathrm{~ms}$ of silence between each word to construct the familiarization and test sequences. The pictures of each referent were photographs of real objects selected from http://clipart.com, edited such that each object appeared by itself on a white background. All items were approximately the same size on the screen (occupying approximately $50 \%$ of the screen, with the white background occupying the other 50\%). To create the familiarization corpora, the three-word sequences were concatenated with $750 \mathrm{~ms}$ of silence between each sequence. Apple QuickTime ${ }^{\mathrm{TM}}$ movies of each sequence were constructed such that a static visual image of the referent on a white background appeared concurrent with the audio presentation of each word. For example, when children heard a familiarization sequence like "dog-towel-kitty", they concurrently saw the same sequence of images, presented one at a time. During the test phase, the spoken test stimuli were not accompanied by pictures of referents. Instead, the same neutral attention-getting visual stimulus (a rotating pinwheel) was used on all test trials.

\subsubsection{Procedure}

Toddlers were tested in a two-square-meter sound-attenuated booth equipped with three computer monitors, one in the front center and one on each side, and two audio speakers, placed directly next to the side monitors. Participants were seated in a caregiver's lap; caregivers listened to masking music over closed-ear headphones, and wore blacked-out sunglasses. The experiment was run using a modified version of the Headturn Preference Procedure (62), and consisted of a familiarization phase and a test phase. The familiarization stimuli were presented using Apple QuickTime ${ }^{\mathrm{TM}}$. The test items were presented and controlled using HABIT software (63), under the control of the experimenter sitting outside the booth, watching the child over closed-circuit video. The experimenter was blind to condition and to which stimulus was playing on each trial.

During the familiarization phase, each participant heard and saw the two-minute familiarization corpus. The video for the familiarization corpus was displayed on the front-center monitor, and the audio was played from both the left and right speakers concurrently. After the familiarization phase, participants received two trials designed to familiarize them with stimuli appearing on the sides of the booth. On these trials, an attention-getting stimulus appeared on the center monitor (a rotating pinwheel), accompanied by music played on both side speakers. When the participant looked at the center stimulus, it disappeared and was replaced by a stimulus on either the left or right side monitor (the same rotating pinwheel) accompanied by music on the side the visual stimulus was playing. The side stimulus continued to play for 20 seconds or until the participant looked away for more than two seconds, whichever came first. After the two side monitor exposure trials (one on each side), the participant received 16 test trials: two repetitions of the 8 test strings, blocked and randomized within each block. Each of these trials also contained the center attention-getting stimulus, followed by a test stimulus on either the left or the right 
side (audio test string, visual rotating pinwheel). On each trial, a single three-word string was repeated (with a $750 \mathrm{~ms}$ pause between each repetition) for 20 seconds or until the participant looked away for more than two seconds, whichever came first. The entire familiarization phase lasted approximately two minutes, and the test phase lasted approximately two to three minutes.

\subsection{Results and Discussion}

We conducted a $2 \times 2 \times 2$ mixed ANOVA on looking times, with familiarization condition (Semantic Match vs. Control) and child gender as between-subjects factors and test item type (Familiarized vs. Novel) as a within-subject factor. The main effects and interactions involving gender were not significant (all F's $<1$ ). There were also no significant main effects of familiarization condition or test item type (both F's $<1$ ). There was, however, a significant interaction between familiarization condition and test item type $[\mathrm{F}(1,46)=4.39, p<0.05]$. We conducted planned follow-up tests separately for the Semantic Match and the Control conditions, using standard error pooled across both conditions (64). Toddlers in the Semantic Match condition looked significantly longer on Novel trials $(\mathrm{M}=11.5$ sec., $\mathrm{SE}=0.8)$ than on Familiar trials $[\mathrm{M}=10.4$ sec., $\mathrm{SE}=0.8 ; \mathrm{F}(1,23)=4.42, p<0.05]$. However, toddlers in the Control condition did not discriminate between Novel trials $(\mathrm{M}=10.2 \mathrm{sec} ., \mathrm{SE}=0.7)$ and Familiar trials $[\mathrm{M}=10.8 \mathrm{sec} ., \mathrm{SE}=0.6 ; \mathrm{F}(1,23)=0.83, p=0.37]$. See Figure 1 for group data and scatterplots depicting the mean scores for individual toddlers.
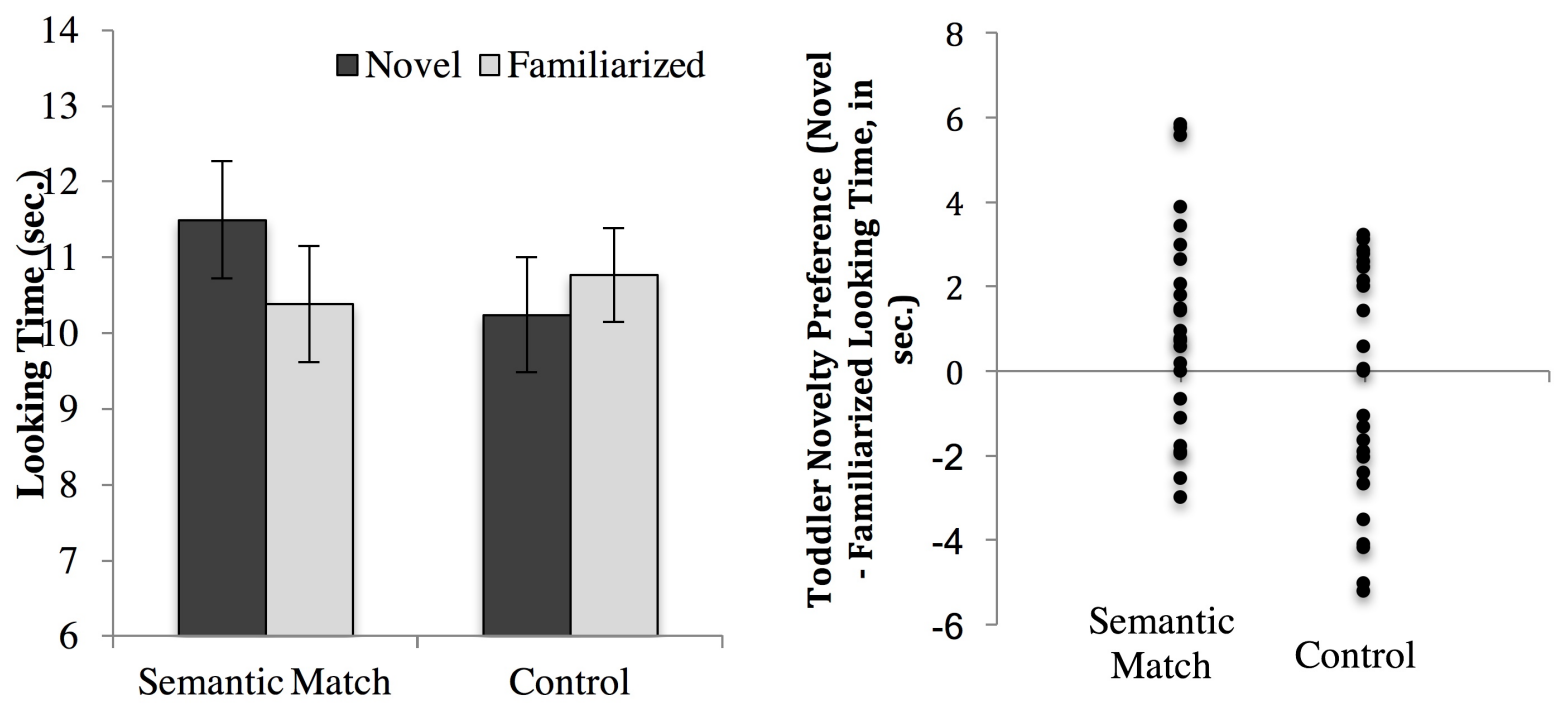

Fig. 1. Average looking times on Familiar and Novel test trials in Experiment 1, as a function of training condition (Semantic Match versus Control). Left: mean and standard deviations in each condition. Right: Scatterplots showing the Novelty preferences for each participant as a function of familiarization condition.

Experiment 1 confirms that 24-month-olds show facilitated learning of nonadjacent dependencies when a semantic cue is aligned with the nonadjacent structure. Toddlers in the Semantic Match condition, who heard a familiarization corpus containing nonadjacent pairs drawn from the same taxonomic category, successfully discriminated between familiarized test strings that were consistent with the nonadjacent grammar and novel strings that were inconsistent. Toddlers' failure to show discrimination in the Control condition replicates findings by Gómez (2002), Gómez and Maye (2005), and Newport and Aslin (2004), and provides further evidence that learning lexical nonadjacent dependencies is difficult when there is no grouping cue or attention-directing contingency pointing learners to reliable dependencies in the input. Toddlers' failure to show discrimination in the Control condition also 
eliminates a number of other possible explanations for success in the Semantic Match condition, including the age of the participants, the presence of highly familiar words, and the addition of visual referents during the familiarization phase.

The results of Experiment 1 demonstrate that toddlers discriminate between familiarized strings containing nonadjacent dependencies and novel strings that violate those dependencies when there is an aligned semantic cue. One possible explanation for this finding is that the aligned semantic cue (or the semantic overlap) made it easier for children to memorize the familiarization strings; in other words, the string "dog-towel-kitty" was easier for the children to remember than "dog-towel-banana." This possibility is consistent with research demonstrating that adults have an easier time learning sequences with internal semantic structure $(48,65-66)$. The ability to memorize strings containing semanticallyorganized nonadjacent dependencies may be an important first step in learning nonadjacent structure. But to develop the kind of sensitivity to nonadjacent dependencies that would assist in the productive use of language, children must go beyond memorizing specific strings, generalizing the dependency to include new stimuli. In Experiment 2, we asked whether toddlers could transfer their experience with a nonadjacent dependency to test strings in which the familiar nonadjacent elements co-occurred with a new intervening $\mathrm{x}$-item.

\section{Experiment 2}

This study was designed to determine whether toddlers presented with nonadjacent linguistic structure that is correlated with semantic cues (as in the Semantic Match condition from Experiment 1) can transfer that knowledge to novel strings containing the nonadjacent pair. To address this question, we changed the intervening $\mathrm{x}$-items between familiarization and test. Toddlers were familiarized with strings containing the nonadjacent pairs "dog-x-kitty" and "cookie-x-banana" with one set of intervening x items (shoe, phone, \& keys). The test items contained new intervening items (e.g., "dog-towel-kitty" vs. "dogtowel-shoe"). If toddlers fail to discriminate between the test items in Experiment 2, the results of Experiment 1 should be interpreted as suggesting that semantically-aligned cues facilitate memory for specific items. However, if toddlers in Experiment 2 successfully discriminate between Familiar and Novel strings given new $\mathrm{x}$-items, this result would provide evidence that the toddlers learned about the nonadjacent dependency itself (e.g., "dog-x-kitty"), and can recognize that construction despite the presence of a new intervening word. As in Experiment 1, we included both Semantic Match and Control conditions; the only change was the use of different $\mathrm{x}$-items in the training and test stimuli.

\subsection{Method}

\subsubsection{Participants}

Forty-eight toddlers (24 females) from English-speaking families participated in the study (mean age 24.2 months; range: 22.2 to 25.6 months) and were randomly assigned to one of the two different familiarization conditions (Semantic Match condition: 12 females, mean age 23.9 months; Control condition: 12 females, mean age 24.1 months). All of the participants were typically developing and fullterm according to parental report. Three additional toddlers were tested but did not complete the experiment due to fussiness.

\subsubsection{Materials and Procedure}

The materials, procedure, and design for Experiment 2 were identical to Experiment 1, with one important change: the intervening $\mathrm{x}$-items in the familiarization and test strings consisted of different words. During familiarization, the intervening x-words were key, phone, and shoe. During the test phase, the $\mathrm{x}$-words were comb and toothbrush (identical to Experiment 1). The full set of strings is shown in Appendix 1. 


\subsection{Results and Discussion}

As in Experiment 1, we conducted a 2x2x2 mixed ANOVA on looking times, with familiarization condition (Semantic Match vs. Control) and child gender as between-subjects factors and test item type (Familiarized vs. Novel) as a within-subject factor. In Experiment 2, no main effects were significant; the two-way interaction between gender and test-item type was also not significant. However, there was a significant interaction between gender and training condition $[\mathrm{F}(1,63)=8.36, \mathrm{p}=0.006]$, as well as a two-way interaction between training condition and test item type $[\mathrm{F}(1,62)=4.33 \cdot \mathrm{p}=0.043]$. There was also a significant three-way interaction $[F(1,46)=19.75, p<0.001]$. Follow-up analyses revealed no significant effects for either boys or girls in the Control condition (boys: $t(11)=1.40, p=0.19$; girls: $t(11$ ) $=0.39, p=0.71]$. There were, however, significant differences for both genders in the Semantic Match condition. The boys showed a significant familiarity preference, with longer looking times for familiar items $(\mathrm{M}=12.84, \mathrm{SE}=0.76)$ than novel items $[\mathrm{M}=9.27, \mathrm{SE}=0.56 ; \mathrm{t}(11)=4.61, \mathrm{p}<0.001]$. In contrast, the girls showed a significant novelty preference, with longer looking times for novel items $(\mathrm{M}=11.40$, $\mathrm{SE}=1.12$ ) than familiar items $[\mathrm{M}=10.15, \mathrm{SE}=1.32 ; \mathrm{t}(11)=2.45, \mathrm{p}=0.032]$. Figure 2 presents group data for Experiment 2, broken down by condition and gender. Figure 3 depicts novelty preference scores for individual toddlers as a function of gender and training condition.

When the test materials required participants to generalize to new $\mathrm{x}$-items, toddlers only showed evidence of learning in the presence of a relationship between the nonadjacent dependency and a semantic relation, just as observed in Experiment 1 . However, the direction of the effect (familiarity versus novelty preference) varied by participant gender: boys showed a familiarity preference and girls showed a novelty preference. As shown in Figure 3, the number of males showing a familiarity preference and the number of females showing a novelty preference were comparable (11 of 12 males and 10 of 12 females showed their respective effects).

Experiment 2 extends the findings from Experiment 1 by showing that the facilitating effects of semantically related nonadjacent elements extend beyond simple string memorization. Rather, correlated semantic structure appears to help toddlers acquire generalizable knowledge about nonadjacent constructions such as "dog-x-kitty." After hearing exemplars like "dog-shoe-kitty" and "dog-keys-kitty", toddlers were able to transfer that knowledge to novel strings such as "dog-towel-kitty." Initial experience with a semantically-congruent nonadjacent dependency facilitated the processing of new stimuli containing that dependency.

The opposite pattern of preferences shown by male and female toddlers in Experiment 2 is consistent with previous research demonstrating gender differences in language acquisition. Females routinely have higher vocabulary scores on child parental reports of vocabulary acquisition (59). Likewise, in behavioral studies of language and pattern acquisition, females sometimes show experimental evidence of learning when males do not $(37,67,68)$, and are more likely to generalize abstract linguistic knowledge (69).

The present findings are interpretable within this context of gender-correlated developmental differences. In studies that use preferential looking as a dependent measure, preferences for familiar (or familiarized) vs. novel items are believed to reflect the difficulty of learning or processing the stimuli (7073). If toddlers have an easy time encoding a new stimulus or learning a new pattern, or already have considerable knowledge about a particular stimulus, they will habituate to that stimulus more easily, and are more likely to show a novelty preference. If instead the new stimulus is more complex and more difficult to learn, or the infant has relatively little starting knowledge in the domain, they will be more likely to show a familiarity preference, perhaps signifying they have just noticed or are currently interested in the familiarization pattern, and require additional experience to habituate. For example, in a semantic priming task, infants showed a familiarity preference when the words were newly acquired, but a novelty preference when the words were already well known $(61,74)$.

Extending this framework to the current design, we would expect toddlers to show a novelty preference if they were able to learn the nonadjacent dependency and map it to the test items relatively easily. We would expect a familiarity preference if they were able to learn the nonadjacent dependency, 
but with difficulty. And we would expect no difference between the conditions if they were not able to learn the nonadjacent dependency at all. For toddlers in the Control condition of Experiments 1 and 2 (the hardest condition across both experiments), we observed no significant preference in either direction, and no gender differences. Without correlated semantic cues, toddlers did not learn the nonadjacent dependency (Experiment 1) or transfer knowledge of that dependency to new strings (Experiment 2).

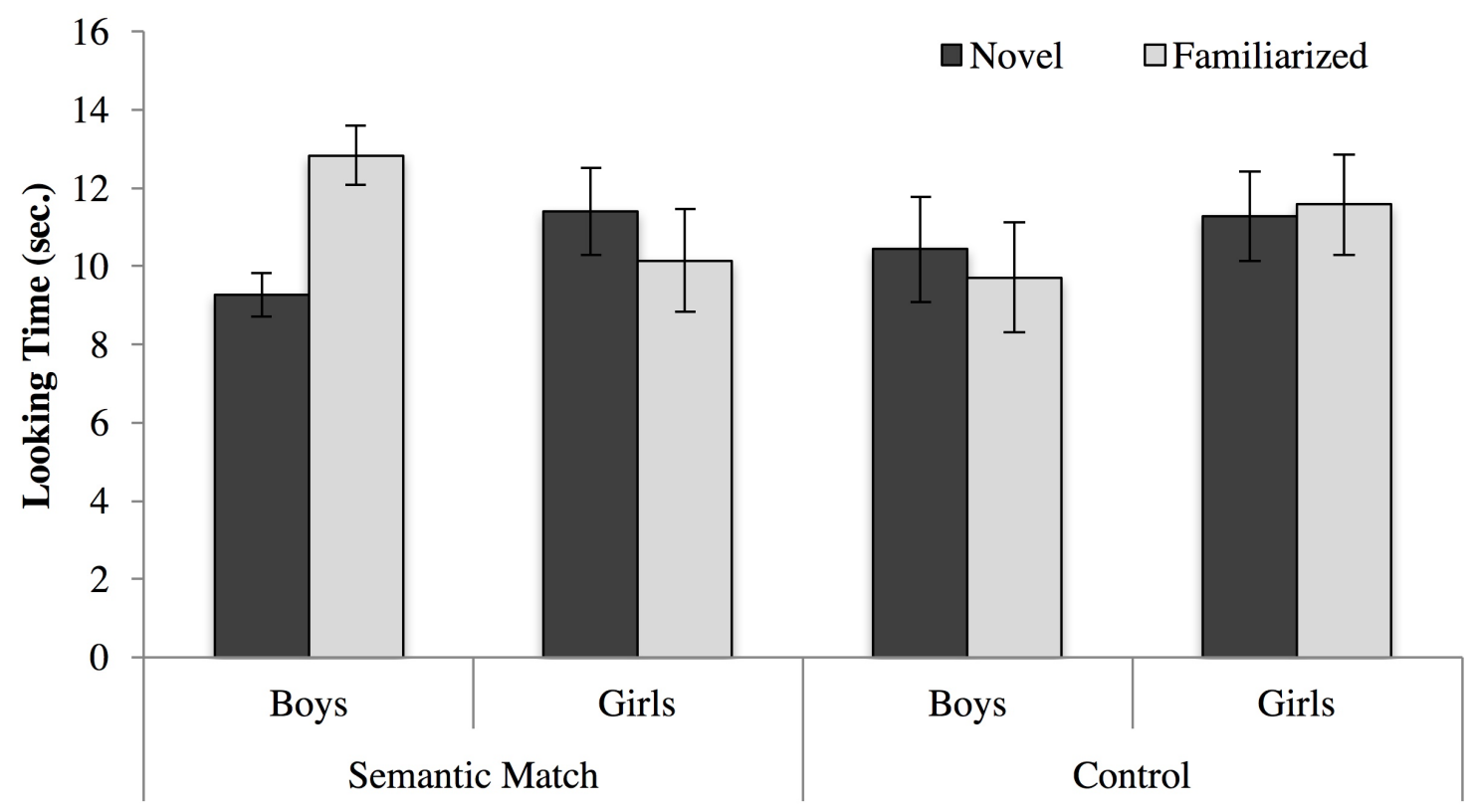

Fig. 2. Average looking times on Familiar and Novel test trials in Experiment 2, split by gender and training condition.

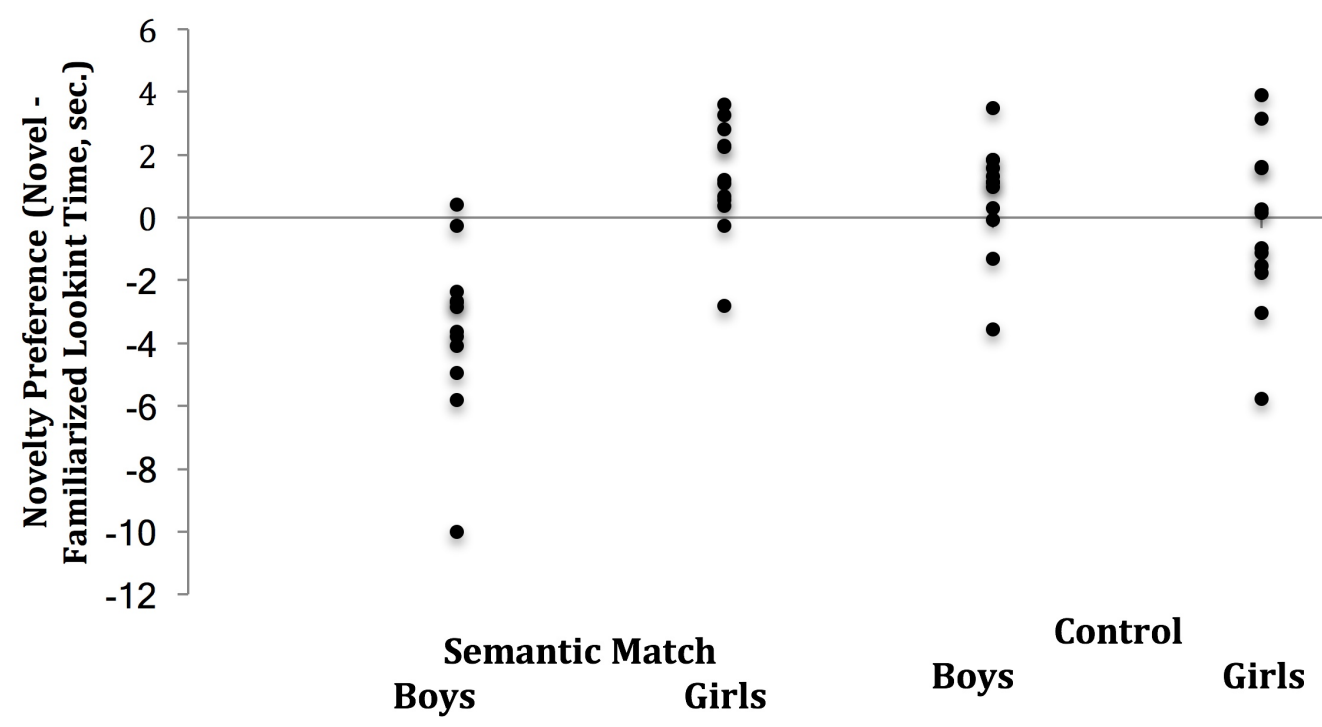

Fig. 3. Scatterplots showing the Novelty preferences for individual subjects in Experiment 2 (looking times on Novel trials minus their looking times on Familiar trials) as a function of gender and training condition. 
For toddlers in the Semantic Match condition of Experiment 1 (the easiest condition across both experiments), we observed a significant novelty preference and no gender differences. In this experiment, both males and females learned the dependency and did so relatively easily. In the Semantic Match condition of Experiment 2 (which involved transfer to new x-items), toddlers of both genders still successfully learned the nonadjacent structure and transferring it to novel strings, but they exhibited this discrimination effect in opposite directions. Female toddlers continued to exhibit a novelty preference, suggesting that they found this potentially difficult transfer task to be relatively easy. In contrast, male toddlers exhibited a familiarity preference, suggesting that the transfer task was relatively challenging for them. This difference, favoring female toddlers, aligns with the previously cited research indicating that females are slightly more advanced in language development, especially on tasks involving transfer of abstract linguistic knowledge (69) and in learning nonadjacent dependencies $(36,68)$.

Regardless of the specific mechanisms underpinning the gender interaction, both genders showed discrimination between novel and familiarized test strings in the Semantic Match condition, but not in the Control condition. Learning does not appear to be bound to the specific three-word strings that toddlers encountered during training. These results cannot prove that the toddlers were learning an item-specific nonadjacent dependency such as doggie-X-kitty (as opposed to a more abstract relationship such as ANIMAL-X-ANIMAL), they suggest that correlated semantic cues help toddlers notice regularities within linguistic strings, suggesting a possible mechanism whereby learners can begin bootstrapping these dependencies. The subsequent two experiments were designed to further explore the mechanisms underpinning these findings. How do semantic cues facilitate learning? If the category level relationship (or some feature-based correlate of the category) is itself what is driving the learning, then perhaps infants are learning something more abstract, such as relationships between categories and the linguistic structures in which they appear.

\section{Experiment 3}

Experiments 1 and 2 established that toddlers show facilitated learning of associations between nonadjacent items when those items share a correlated semantic cue, and can transfer this knowledge to discriminate between sequences that involve novel intervening items. The next experiment was designed to delve more deeply into the nature of the non-adjacent dependency learning itself. Are toddlers learning a relation between specific words, or are they instead learning a relationship at a higher level of abstraction, such as ANIMAL-X-ANIMAL or FOOD-x-FOOD. Previous research demonstrated that adults show facilitated learning of rule-based sequences such as $\mathrm{ABA}$ or $\mathrm{ABB}$ when the $\mathrm{A}$ and $\mathrm{B}$ items belong to specific semantic categories (41). In our design, since shared category membership is a correlated cue that may help the infants learn the dependency, it may be that infants are actually learning about nonadjacent sequences of categories, rather than nonadjacent sequences of words.

In Experiment 3, we investigated whether learning occurred at the category level by changing the $\mathrm{A}$ and $\mathrm{B}$ items between familiarization and test, within the same semantic category. Thus, toddlers were familiarized with sequences like birdy-X-fish and toast-x-cheese, and tested on dog-x-cat vs. dog-xbanana. If participants learned only the specific association between birdy and fish, they should not succeed on this test discrimination. However, if participants learned something more abstract (such as an association between the categories to which the words belong), we would expect to observe successful discrimination between the familiarized and novel items.

\subsection{Method \\ 4.1.1. Participants}

Forty-eight toddlers (24 females) from English-speaking families participated in the study (mean age 24.2 months; range: 22.1 to 25.5 months) and were randomly assigned to one of the two familiarization conditions (Semantic Match condition: 12 females, mean age 24.0 months; Control condition: 12 females, mean age 24.1 months). All of the participants were typically developing and full- 
term according to parental report. Three additional toddlers were tested but did not complete the experiment due to fussiness.

\subsubsection{Materials and Procedure}

The design and procedure for Experiment 3 were identical to Experiments 1 and 2. The test items for Experiment 3 were the same as Experiment 1 (doggie-x-kitty, cookie-x-banana, doggie-x-banana, cookie-x-kitty, with the $\mathrm{x}$-items soap and toothbrush). However, the familiarization A-B pairs were different items from the same semantic categories: bird-x-fish and toast-x-cheese. Effectively, the training materials were identical to Experiment 1 as described in Table 1, but with birdie, fish, toast, and cheese substituted for doggie, kitty, cookie, and banana, respectively. The same $\mathrm{x}$-items were used for familiarization and test. The full set of strings is shown in Appendix 1.

\subsection{Results and Discussion}

We conducted a $2 \times 2 \times 2$ mixed ANOVA on looking times, with familiarization condition (Semantic Match vs. Control) and gender as between-subjects factors, and test item type (Familiarized vs. Novel) as a within-subject factor. The results of Experiment 3 were similar to Experiment 1, with no effect of gender on performance. The only significant effect was the two-way interaction between training condition (Semantic Match vs. Control) and test item type (familiarized vs. novel): $\mathrm{F}(1,46)=4.52, p=$ 0.039. Follow-up tests revealed a significant difference in the Semantic Match training condition, with longer looking times on novel trials $(\mathrm{M}=13.67, \mathrm{SE}=0.51)$ compared to familiar trials $[\mathrm{M}=12.40, \mathrm{SE}=$ $0.57 ; t(23)=2.63, p=0.015]$. There was no such difference in the control condition, with similiar looking times in the novel trials $(\mathrm{M}=11.32, \mathrm{SE}=0.82)$ and familiar trials $[\mathrm{M}=11.42, \mathrm{SE}=0.87 ; t(23)=0.23, p=$ $0.821]$. Thus, only learners in the Semantic Match condition successfully generalized to novel exemplars from the same categories. Figure 4 depicts looking times for Experiment 3 (group and individual data) as a function of training condition and test item type.
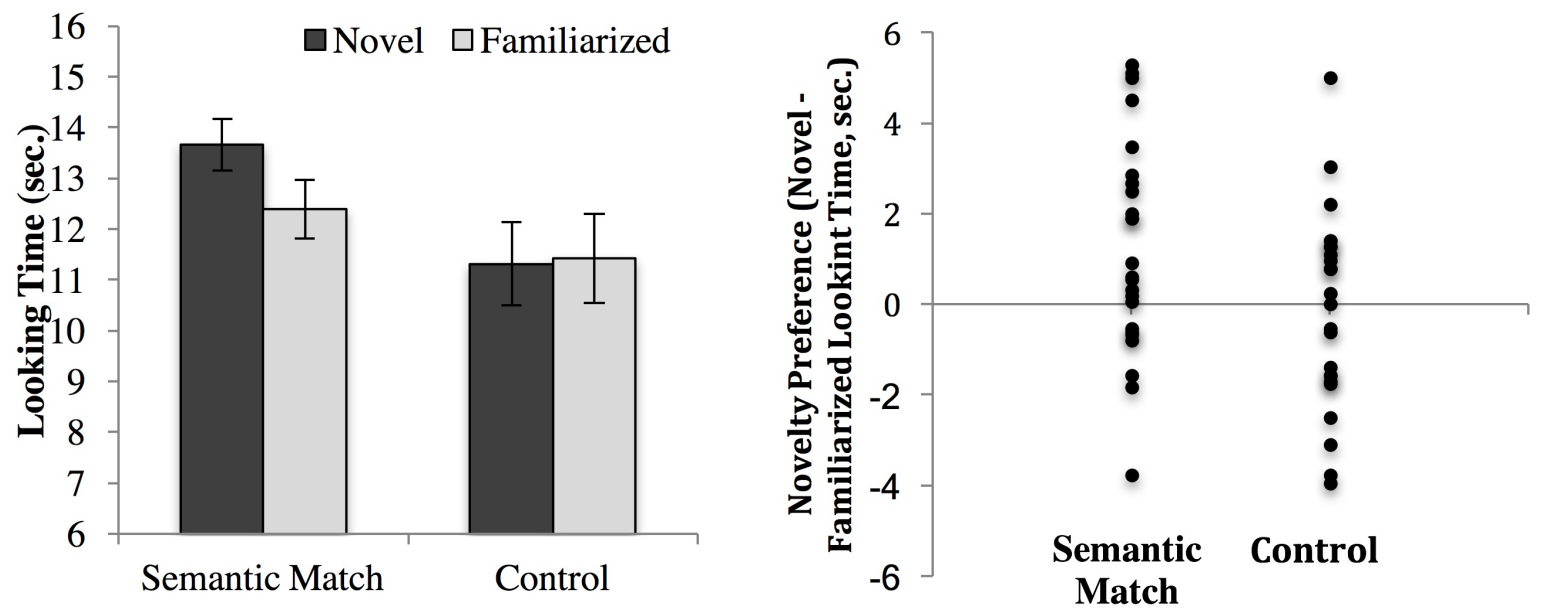

Figure 4. Average looking times on Familiar and Novel test trials in Experiment 3, as a function of training condition (Semantic Match versus Control). Left: mean and standard deviations in each condition. Right: Scatterplots showing the Novelty preferences (looking times on Novel trials minus their looking times on Familiarized trials) for each child.

These results are helpful for understanding what toddlers are learning in this series of experiments. In Experiments 1 and 2, participants in both the Semantic Match and Control conditions were presented with audio-visual sequences following an AxB grammar, where each A-item perfectly predicted each B-item. In both experiments, they only demonstrated learning in the Semantic Match conditions, when the A and B items were semantically similar. There are a number of possible mechanistic explanations for this finding (more than we can eliminate in a single paper). But one 
possible explanation is the mechanism suggested by Newport and Aslin (2004): the similarity relationship between non-adjacent items draws attention to them, which then boosts learning of their association. This mechanism may be complemented by the fact that children may not necessarily be learning an association between the specific items at all, but are instead learning about relations at a more abstract level, such as between the categories to which the words belong, as previously observed in adults (41).

The results from Experiment 3 provide evidence that this latter mechanism may be part of the explanation. Toddlers who were trained on the sequences birdy-X-fish and toast-X-cheese discriminated between novel items consistent with the trained sequence of categories (e.g., doggy-x-kitty) versus items inconsistent with the trained sequence of categories (e.g., doggie-x-banana). These data suggest that the toddlers were learning something quite abstract, such as an association at the level of the category (e.g. ANIMAL-X-ANIMAL and FOOD-X-FOOD). Notably, many lexical nonadjacent dependencies exist as relationships between linguistic structures and semantic categories (such as ditransitive verbs and the relationships amongst their nouns). The results of this experiment demonstrate that children are capable of experiencing input containing regularities between specific items, and using that to learn generalized abstract knowledge about mappings between linguistic strings and correlated semantic categories. This is a useful skill that may help them acquire many of the more abstract structures and relations in language.

A final question of interest involves whether the knowledge children are learning about these dependencies are so abstract as to be independent of any of the items that are present when children learn the dependency. In Experiment 2, we altered the intervening x-items between training and test, and in Experiment 3, we altered the specific items comprising the AB-pairs. In Experiment 4, we altered all three elements, resulting in zero lexical overlap between training and test. If toddlers are truly learning an abstract relation between categories and the structure in which they are used in linguistic strings, then they ought to be able to show learning even under these complex conditions.

\section{Experiment 4}

In Experiment 4, we concurrently included both of the transfer manipulations from Experiments 2 and 3, altering both the $\mathrm{AB}$-pairs and the intervening $\mathrm{x}$-items between training and test. This manipulation is interesting for two reasons. First, the training and test items contain zero overlap in terms of specific lexical items. If the toddlers discriminate between novel and familiarized items in Experiment 4, it cannot be on the basis of any particular words or item-specific associations, but instead must be a function of the correlations among the more abstract semantic relations and the linguistic structure. Second, Experiment 4 provides an opportunity to attempt to replicate the gender interaction in Experiment 2, which we did not observe in Experiment 3. The gender interaction in Experiment 2 (particularly boys' relative difficulty, demonstrated by their familiarity effect) suggests that the transfer performed in Experiment 2 was more difficult than the transfer performed in Experiment 3 (where both genders showed a novelty effect). Experiment 4 - in which all test items are novel relative to the training items - should be the most difficult task of all. If we again observe flips in direction of preference, we can be more confident that the gender interaction observed in Experiment 2 was not spurious.

\subsection{Method}

\subsubsection{Participants}

Forty-eight toddlers (24 females) from English-speaking families participated in the study (mean age 24.2 months; range: 22.0 to 25.8 months and were randomly assigned to one of the two different familiarization conditions (Semantic Match condition: 12 females, mean age 23.9 months; Control condition: 12 females, mean age 24.0 months). All of the participants were typically developing and fullterm according to parental report. Three additional children were tested but did not complete the experiment due to fussiness. 


\subsubsection{Materials and Procedure}

The design and procedure for Experiment 4 were identical to Experiments 1-3, and included both the $\mathrm{x}$-item training-test manipulation from Experiment 2 and the AB-item training-test manipulation from Experiment 3. The full set of items is shown in Appendix 1.

\subsection{Results and Discussion}

We conducted a $2 \times 2 \times 2$ mixed ANOVA on looking times, with familiarization condition (Semantic Match vs. Control) and gender as between-subjects factors, and test item type (Familiarized vs. Novel) as a within-subject factor. The results in Experiment 4 were comparable to Experiment 2. There were no significant main effects, and there was a significant two-way interaction between gender and training condition $[\mathrm{F}(1,47)=8.48, \mathrm{p}=0.006]$. As in Experiment 2, there was also a significant three-way interaction $[\mathrm{F}(1,46)=4.11, \mathrm{p}=0.047]$. Follow-up analyses revealed a pattern qualitatively similar to Experiment 2. In the Control condition, neither gender successfully discriminated between novel and familiar test trials [boys: $t(1,11)=0.38, p=0.71$; girls: $t(1,11)=0.56, p=0.59$ ]. In the Semantic Match condition, the results mirrored Experiment 2: boys showed significantly longer looking times on familiarized test trials $(\mathrm{M}=11.87, \mathrm{SE}=0.67)$ compared to novel test trials $(\mathrm{M}=10.65, \mathrm{SE}=0.70 ; \mathrm{t}(1,11)$ $=2.31, \mathrm{p}=0.042 \mathrm{]}$; girls showed significantly longer looking times on novel test trials $(\mathrm{M}=11.15, \mathrm{SE}=$ $0.96)$ compared to familiarized test trials $(\mathrm{M}=8.94, \mathrm{SE}=0.69 ; \mathrm{t}(1,11)=2.53, \mathrm{p}=0.028]$. Figure 5 depicts the group looking times for Experiment 4, broken down by test trial type, condition and gender. Figure 6 shows individual toddlers' novelty preferences as a function of gender and training condition.

Experiments 1-4 can be combined into a 5-factor design with test trial type (novel versus familiarized) manipulated within subjects (using the exact same test items across all conditions), and four between-subjects factors: gender, training condition (semantic match vs. control), AB-item condition (same versus different at training and test), and X-item condition (same versus different at training and test). A five-way ANOVA conducted on the data from all four experiments yielded no significant effects or interactions involving the AB-item manipulation. In other words, whether or not the AB items were the same at training and test did not have a significant effect on looking time alone or interacting with other variables. However, the four-way interaction between the other variables was significant: $\mathrm{F}(1,176)=5.42$, $p=0.021$. This four-way interaction summarizes the findings across the experiments. Looking times to novel versus familiar test trials was significantly affected by whether the training condition included nonadjacent dependencies with correlated semantic cues. Toddlers showed evidence of learning only when there was a correlated semantic cue. However, this effect was modulated by whether the intervening items were the same at training and test and the child's gender. If the $\mathrm{x}$-items were the same, both genders showed a novelty preference. However, if the x-items were different (a more difficult task involving transfer to novel items), then girls showed a novelty preference while boys showed a familiarity preference.

Experiment 4 demonstrates that when there was a semantic cue correlated with the nonadjacent structure, toddlers of both genders discriminate between novel and familiarized test trials and thus show evidence of learning the regularity, facilitated by correlated semantic structure. Replicating the gender interaction in Experiment 2, boys' discrimination came in the form of a familiarity effect, whereas girls demonstrated a novelty effect. Thus, given challenging transfer-based tasks, the gender interaction implies that girls learn more easily than boys, consistent with prior research $(36,68,69)$. As those papers note, it is unlikely that gender per se is the causal variable. Instead, gender is correlated with other factors strongly enough to impact the results of studies with different aged children, stimuli and methodologies (i.e. girls show a novelty effect while boys show a familiarity effect). These findings also align with other studies showing girls ahead in various language measures at these ages (such as the MCDI norms, 59). Future research will investigate whether these vocabulary advantages are the causal explanation, as considerable research demonstrates numerous facilitative effects of high vocabulary on downstream learning $(75,76)$. However, it is also possible that some lower level or mechanistic cause (such as 
memory or attention differences) facilitates vocabulary acquisition in general, as well as the ability to learn nonadjacent structure in these experiments.

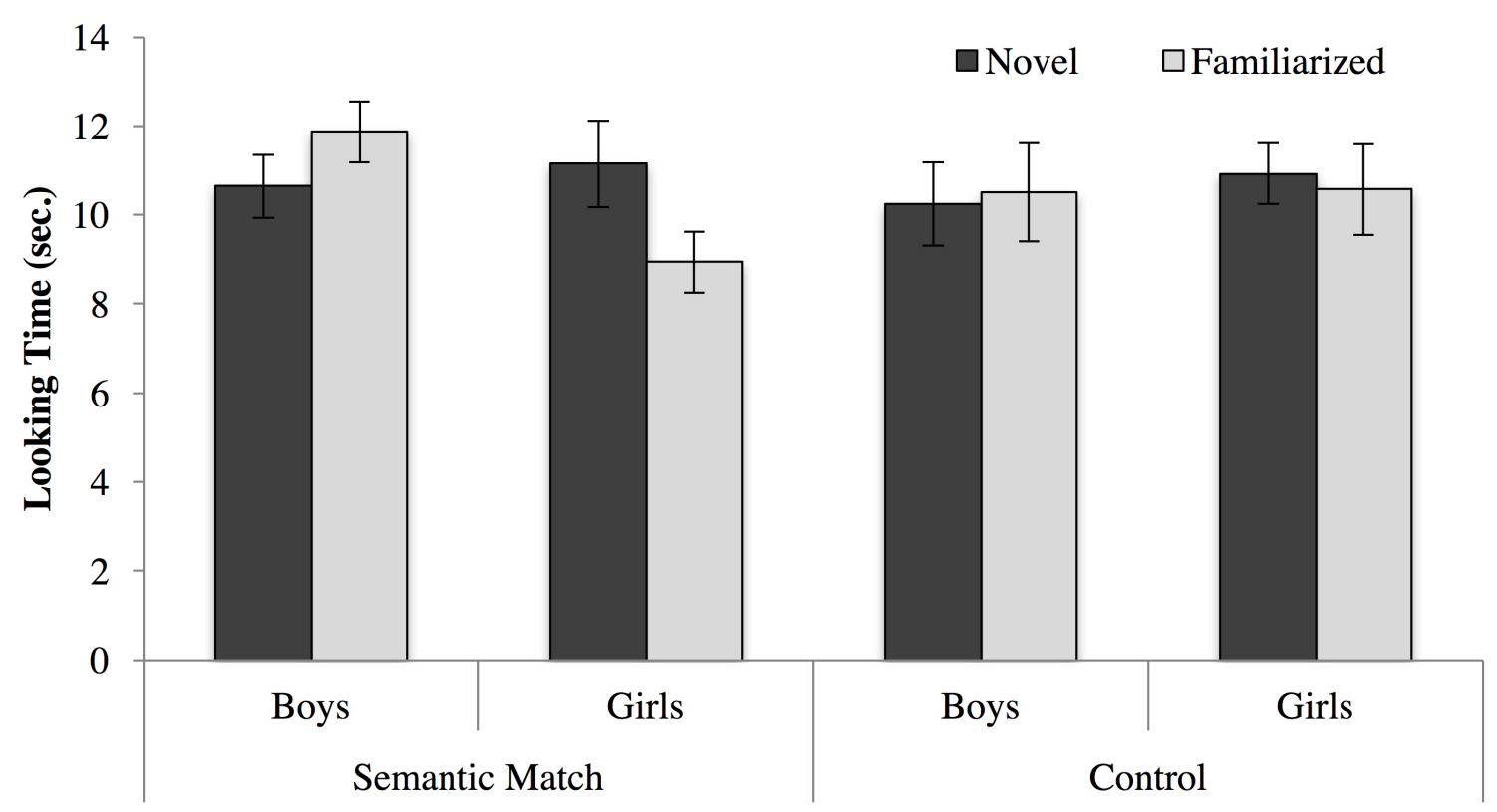

Figure 5. Average looking times on Familiar and Novel test trials in Experiment 4, broken down by gender and training condition.

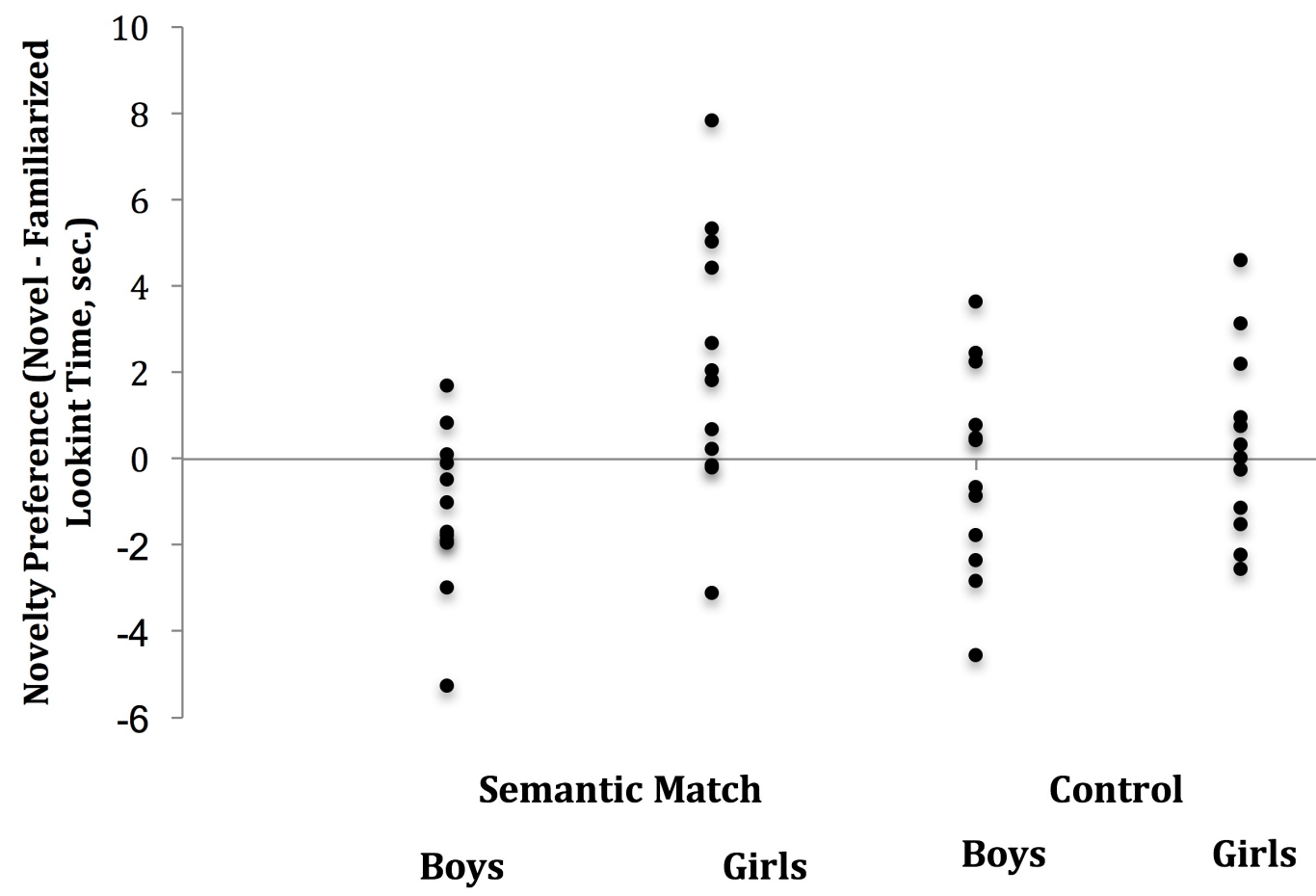

Figure 6. Scatterplots showing the Novelty preferences for individual subjects in Experiment 4 (looking times on Novel trials minus their looking times on Familiar trials) as a function of gender and training condition. 


\section{General Discussion}

There is ongoing debate over the mechanisms by which humans acquire knowledge about language's complex dependencies, and the acquisition of nonadjacent structure has played a key role in this debate. On the one hand, nonadjacent dependencies are a critical feature of human language, and infants, toddlers, and adults demonstrate extensive knowledge and use of nonadjacent structure in natural languages. On the other hand, learners do not readily acquire novel lexical nonadjacent dependencies in laboratory situations, especially under conditions simulating some of the more complex and abstract nonadjacent dependencies present in natural languages, where no cues to that relationship are present.

However, it has in many of these real-world situations, there are external cues correlated with the nonadjacent dependencies, raising the possibility that these cues may facilitate the acquisition of an otherwise difficult distributional pattern. Endress and colleagues $(26,77)$ have demonstrated in a number of experiments that adults achieve facilitative learning of nonadjacent dependencies for units that exist at utterance boundaries. These units seem to be treated differently (perhaps due to increased attention due to their salience, perhaps due to the engagement of distinct learning mechanisms for these units). Van den Bos et al. (2012), Newport and Aslin (2004), and Turk-Browne et al (2008) among others have demonstrated facilitated learning of nonadjacent dependencies when they are supported by correlated perceptual cues $(27,39,78)$.

In the current work, we demonstrated that facilitated learning is also achieved in the present of correlated semantic cues, a situation with potential relevance to a number of lexical nonadjacent dependencies that children need to learn. In Experiment 1, toddlers successfully learned a novel grammar containing nonadjacent dependencies only when the semantic structure was correlated with the nonadjacent structure. In Experiment 2, toddlers showed evidence of learning a construction like dog-xkitty, which they can transfer to include novel $\mathrm{x}$-items, but again only when the semantic structure was correlated with the nonadjacent structure. In Experiment 3, toddlers familiarized on a nonadjacent sequence like birdy-x-fish transferred that knowledge to strings like dog-x-cat, providing evidence that toddlers learned about the sequential relationships amongst categories (or perhaps about the relation of the semantic features or associations that those words share). In Experiment 4, toddlers who learned about a nonadjacent sequence transferred that knowledge to strings that shared no lexical overlap with the trained strings, suggesting that the learned patterns were more abstract than the specific item sequences heard during training.

These experiments suggest a learning mechanism whereby toddlers may be able to exploit correlated semantic cues when learning nonadjacent dependencies. Obviously, these materials were far more simple and artificial than the structures observed in natural languages. Nevertheless, semantic cues are often correlated with linguistic structures in real languages. For example, when learning which nouns are likely to be arguments of a newly learned verb, those arguments frequently have a number of semantic properties in common, including membership in the same semantic or grammatical category (4), shared features (79), frequent co-occurrence (both in language and in the world, Willits, Amato, \& MacDonald, 2016,80 ), and jointly and consistently participating in the same contexts (again, both in language and in the world; $57,58,81$ ).

The current research is consistent with and extends previous research and theories about nonadjacent dependency learning. Multiple studies have provided evidence that adults learn a nonadjacent structure when a perceptual cue is correlated with the nonadjacent structure $(27,32,39,78)$. Our research shows that this process is not limited to perceptual cues, and that by including semantic or conceptual relations, a larger range of nonadjacent dependencies may be learnable using a similarity-bootstrapping mechanism. Our findings support work by others suggesting that adults show facilitated learning of nonadjacent structure in the presence of semantic relations $(39,41,82)$. The findings are also consistent with prior research showing that children and adults can more easily learn a nonadjacent dependency if they have previous experience with an adjacent association between those elements $(36,68)$. In the current experiments (and in many natural language situations involving nonadjacent dependencies), the 
fact that the referents of the words may be "adjacently" associated in semantic memory may make the nonadjacent-dependency learning task considerably easier.

Given the current evidence suggesting that conceptual grouping facilitates the acquisition of nonadjacent dependencies, along with prior work suggesting that this is also true of perceptual grouping cues, it is conceivable that any kind of similarity detected by our cognitive system may provide a potential grouping cue. As such, it is worth considering other known examples of nonadjacent dependency learning, and seeing whether they might also be recast as examples of learning based on similarity-driven grouping cues. One possible example is the set of studies by Peña et al. (1999), Marcus et al. (1999), and Endress et al. (2005) $(26,30,43)$. As noted by Endress et al., successful learning in these studies seems to have turned on whether or not the nonadjacently related pair is on the edge of the sequence (i.e., the first and the last element in a sequence). One interpretation of this observation is that the nonadjacent elements in these studies may be learnable because they can be grouped in terms of their similarity: both co-occur with pauses at the utterance boundaries. Likewise, one possible mechanism underlying Gómez's (2002) set size finding - that adults and infants can learn nonadjacent dependencies if the set size of the intervening words is disproportionally much larger than the set size of the nonadjacently related words is a form of similarity-based grouping, in terms of the similarity of the set sizes that make up the different slots in the construction (23). And indeed, follow-up research has shown that if the set size of the intervening words is also very small (e.g., one item), nonadjacent dependency learning is easier (29). This result is explained nicely by a similarity-based account but requires a second, separate mechanism when explained under the rubric of the set-size effect.

Many questions remain about the exact mechanisms underlying the effects in the current experiments. A number of different learning, memory, and attention-based explanations could explain these results, and future work (both behavioral and computation modeling) is planned to answer these questions. One issue regards the extent to which these effects are driven by the fact that the nonadjacent dependencies are edge-adjacent (e.g. the relations were amongst the first and last items in the string). Endress et al. $(24,26)$ found that learning nonadjacent sequences is extremely difficult if they are not at sequence edges (though other studies with different materials find learning of non-edge nonadjacent dependencies with extremely large amounts of training, e.g., 83). Peña et al. (30) have suggested that edge-adjacent circumstances may activate a different computational mechanism that is specially attuned to look for this kind of structure. One such possible mechanistic explanation is memory-based, and a consequence of primacy and recency effects. If items at the beginning and end of sequences persist in memory for longer periods of time, they may be accessible by different computational mechanisms, which may result in different or better learning than items not on sequence edges. And indeed, there is evidence that by six months of age, infants are already showing standard primacy and recency effects (84). However, the entire story cannot be about edge-related memory mechanisms. If edge boundaries and primacy and recency effects were sufficient to learn the structures, the toddlers would have learned in our control conditions, and they did not. Thus, there is clearly something important about the interaction of the memory mechanism and the content that is being encoded in memory.

The same list learning literature provides an explanation consistent with our explanations, namely that semantic structure facilitates memory recall in short term recall, long term recall, and paired associated learning paradigms. $(48,85-88)$. However, our findings involve the implicit acquisition of sequential structure, while in these classical memory studies participants are typically given a set of word pairs and explicitly instructed to associate or remember them. The lack of equivalency between these two situations was one of the many reasons that psychologists studying language acquisition became disillusioned with simple learning and memory theories in the first place. Nonetheless, the fact that both these classical studies and implicit learning of sequential structure exhibit semantic facilitation effects is intriguing and raises questions about how these two phenomena might be related. Is nonadjacent dependency learning just a special case of classical learning and memory effects, subject to the same principles, such as semantic facilitation? Or are classical learning and memory phenomena and the learning of abstract structure in linguistic strings fundamentally different phenomena that both capitalize 
on the presence correlated semantic cues? Perhaps more relevant is research in list memory, which also exhibits a number of effects of facilitation in the case of semantic relatedness (89-92). A large number of memory models have attempted to capture and explain these effects of interaction between semantic structure, memory, and learning (93-95). Future computational modeling work can see which of these models might be effective at predicting our results, thus helping clarify some of the mechanistic underpinnings of the effect.

More generally, the current research is consistent with the emerging notion that the demands of acquiring nonadjacent structure are not incommensurate with the power of associative learning mechanisms, and do not require strong assumptions about complex syntactic representational knowledge. Instead, there are a variety of different cues that humans can bring to bear on learning nonadjacent dependencies. Statistical learning mechanisms such as tracking transition probabilities $(96,97)$ may be sufficient for learning a large proportion of nonadjacent dependencies, especially those that can be perceptually grouped $(27,78)$ or semantically grouped (as in the current studies). In fact, recent research suggests that statistical learning abilities are highly correlated with adults' abilities to process and comprehend complex sentences with nonadjacent dependencies. Wells et al. (98) found that participants who were given extensive experience with complex, object-relative clause sentences showed marked decreases in reading times for these constructions, and Misyak, Christiansen, and Tomblin (99) found that individual differences in skill at learning nonadjacent dependencies in an artificial grammar predicted which participants had difficulty comprehending complex, object relative clause sentences.

One open question about the above research concerns the degree of abstractness of the knowledge that has been learned. Our findings are consistent with a number of different specific interpretations. In the first, the toddlers were learning associations between specific words, i.e. that dog predicts cat, and were facilitated in doing so because $\operatorname{dog}$ and cat where semantically similar words. Alternatively, the association could be being made at a more abstract level, that there were sequences like ANIMALBATHROOM-ANIMAL, and that toddlers were either learning that ANIMAL in slot 1 predicts ANIMAL in slot 3 (or perhaps that a repetition rule across slot one in 3 as in Endress \& Hauser, or Marcus et al.,). Future research and further experiments can be used to tease out the level of specificity vs. abstractness that is supporting the learning in this situation.

A final important consideration is the extent to which the similarity-based learning mechanism outlined above requires conceptual knowledge about words' referents in the world, as opposed to the information about semantic similarity that can be inferred from words' linguistic distributions alone. Recent research has revealed that it would be possible to learn semantic categories for words based purely on their lexical co-occurrence distributions, without using non-linguistic conceptual knowledge (57, 58, $100,101)$. Words like dog and cat, and cookie and banana, are indeed similar in terms of properties of their referents and referential situations. But they are also similar in terms of the other words with which they co-occur. The word $d o g$ and the word cat tend to be used in very similar distributional contexts (such as following similar articles and adjectives, and preceding similar verbs). A large amount of experience with language would provide a basis for judging $d o g$ and cat to be similar based on these lexical distributions, even if one had no experience with the words' referents. It is thus plausible that the semantic grouping hypothesis suggested by our results is a potential mechanism for explaining nonadjacent dependency learning using entirely linguistic distributional knowledge. Language learners may be able to use distributional similarity as a grouping cue that facilitates nonadjacent dependency acquisition. Indeed, Lany \& Saffran (102) found that tracking distributional and phonological cues marking word categories promotes toddlers' learning of the semantic properties common to words within such categories.

The current research suggests that toddlers can use semantic cues to learn linguistic nonadjacent dependencies. Because nonadjacent dependencies are commonly accompanied by parallel semantic relationships in natural language, these findings shed light on how toddlers may acquire the nonadjacent dependencies prevalent in natural languages. More broadly, these results suggest that associative learning 
mechanisms, when provided with rich, redundant input, are capable of tracking key aspects of complex linguistic structure.

\section{Acknowledgements}

This research was funded by a grant from NIDCD to JW (F31DC009936-01), grants from NICHD to JL (F32HD057698) and JRS (R37 HD037466), a grant from the James F. McDonnell Foundation to JRS, NIH Training Grant 5T32HD049899 to the University of Wisconsin-Madison, and by an NIH core grant to the Waisman Center (P30HD03352). We would like to thank Maryellen MacDonald and Mark Seidenberg for useful comments and suggestions about the experiments. We would also like to thank members of the Infant Learning Lab (especially Tracy Brookhyser and Rachel Vohnoutka) for their assistance in preparing stimuli, scheduling, and running participants in the experiments.

\section{References}

1. Saffran, JR., \& Kirkham, NZ. Infant Statistical Learning. Annual Review of Psychology. 2017 69:2.1-2.23.

2. Mintz TH. Frequent frames as a cue for grammatical categories in child directed speech. Cognition. 2003 Nov 30;90(1):91-117.

3. Chomsky N. Syntax structures. Mouton and Co., Publishers, The Hague, Netherlands. 1957.

4. Pinker S, Levin B. Lexical and conceptual semantics. 1991.

5. Gertner Y, Fisher C, Eisengart J. Learning words and rules: Abstract knowledge of word order in early sentence comprehension. Psychological science. 2006 Aug;17(8):684-91.

6. Goldberg AE. Constructions: a new theoretical approach to language. Trends in cognitive sciences. 2003 May 31;7(5):219-24.

7. MacDonald MC, Seidenberg MS. Constraint satisfaction accounts of lexical and sentence comprehension. Handbook of psycholinguistics. 2006;2:581-611.

8. Snedeker J, Trueswell JC. The developing constraints on parsing decisions: The role of lexical-biases and referential scenes in child and adult sentence processing. Cognitive psychology. 2004 Nov 30;49(3):238-99.

9. Tomasello, 2002 [no full citation

10. Santelmann LM, Jusczyk PW. Sensitivity to discontinuous dependencies in language learners: Evidence for limitations in processing space. Cognition. 1998 Dec 31;69(2):105-34.

11. Lidz J, Waxman S, Freedman J. What infants know about syntax but couldn't have learned: experimental evidence for syntactic structure at 18 months. Cognition. 2003 Oct 31;89(3):295-303.

12. Arnold JE, Brown-Schmidt S, Trueswell J. Children's use of gender and order-of-mention during pronoun comprehension. Language and Cognitive Processes. 2007 Jun 1;22(4):527-65.

13. Gillespie M, Pearlmutter NJ. Hierarchy and scope of planning in subject-verb agreement production. Cognition. 2011 Mar 31;118(3):377-97.

14. Naigles L, Gleitman H, Gleitman L. Syntactic bootstrapping and verb acquisition. Language and cognition: a developmental perspective. Norwood, NJ: Ablex. 1993.

15. Booth JR, Harasaki Y, Burman DD. Development of lexical and sentence level context effects for dominant and subordinate word meanings of homonyms. Journal of psycholinguistic research. 2006 Nov 1;35(6):531-54.

16. Khanna MM, Boland JE. Children's use of language context in lexical ambiguity resolution. The Quarterly Journal of Experimental Psychology. 2010 Jan 1;63(1):160-93.

17. Simpson GB, Foster MR. Lexical ambiguity and children's word recognition. Developmental Psychology. 1986 Mar;22(2):147.

18. MacDonald MC, Pearlmutter NJ, Seidenberg MS. The lexical nature of syntactic ambiguity resolution. Psychological review. 1994 Oct;101(4):676.

19. Hartshorne JK, Ullman MT. Why girls say 'holded' more than boys. Developmental science. 2006 Jan $1 ; 9(1): 21-32$.

20. Tanenhaus MK, Boland J, Garnsey SM, Carlson GN. Lexical structure in parsing long-distance dependencies. Journal of psycholinguistic research. 1989 Jan 1;18(1):37-50.

21. Bock K, Eberhard KM, Cutting JC, Meyer AS, Schriefers H. Some attractions of verb agreement. Cognitive Psychology. 2001 Sep 30;43(2):83-128.

22. Houston-Price C, Nakai S. Distinguishing novelty and familiarity effects in infant preference procedures. Infant and Child Development. 2004 Dec 1;13(4):341-8. 
23. Gómez R, Maye J. The developmental trajectory of nonadjacent dependency learning. Infancy. 2005 Mar $1 ; 7(2): 183-206$.

24. Endress AD, Bonatti LL. Rapid learning of syllable classes from a perceptually continuous speech stream. Cognition. 2007 Nov 30;105(2):247-99.

25. Endress AD, Hauser MD. Syntax-induced pattern deafness. Proceedings of the National Academy of Sciences. 2009 Dec 8;106(49):21001-6.

26. Fedor A, Varga M, Szathmáry E. Semantics boosts syntax in artificial grammar learning tasks with recursion. Journal of Experimental Psychology: Learning, Memory, and Cognition. 2012 May;38(3):776.

27. Newport EL, Aslin RN. Learning at a distance I. Statistical learning of non-adjacent dependencies. Cognitive psychology. 2004 Mar 31;48(2):127-62.

28. Bonatti LL, Peña M, Nespor M, Mehler J. Linguistic constraints on statistical computations: The role of consonants and vowels in continuous speech processing. Psychological Science. 2005 Jun;16(6):451-9.

29. Onnis L, Monaghan P, Christiansen MH, Chater N. Variability is the spice of learning, and a crucial ingredient for detecting and generalizing in nonadjacent dependencies. In Proceedings of the Cognitive Science Society 2004 Jan 1 (Vol. 26, No. 26).

30. Peña M, Bonatti LL, Nespor M, Mehler J. Signal-driven computations in speech processing. Science. 2002 Oct 18;298(5593):604-7.

31. Peña M, Bonatti LL, Nespor M, Mehler J. Signal-driven computations in speech processing. Science. 2002 Oct 18;298(5593):604-7.

32. Creel SC, Newport EL, Aslin RN. Distant melodies: statistical learning of nonadjacent dependencies in tone sequences. Journal of Experimental Psychology: Learning, memory, and cognition. 2004 Sep;30(5):1119.

33. Greene RL, Crowder RG. Effects of semantic similarity on long-term recency. The American journal of psychology. 1984 Oct 1:441-9.

34. Misyak JB, Christiansen MH. Extending statistical learning farther and further: Long-distance dependencies, and individual differences in statistical learning and language. In Proceedings of the Cognitive Science Society 2007 Jan 1 (Vol. 29, No. 29).

35. Romberg AR, Saffran JR. All together now: Concurrent learning of multiple structures in an artificial language. Cognitive Science. 2013 Sep 1;37(7):1290-320.

36. Lany J, Gómez RL. Twelve-month-old infants benefit from prior experience in statistical learning. Psychological Science. 2008 Dec;19(12):1247-52.

37. Lany J, Gómez RL, Gerken LA. The role of prior experience in language acquisition. Cognitive Science. 2007 May 6;31(3):481-507.

38. Bonatti LL, Peña M, Nespor M, Mehler J. On consonants, vowels, chickens, and eggs. Psychological Science. 2007 Oct;18(10):924-5.

39. van den Bos E, Christiansen MH, Misyak JB. Statistical learning of probabilistic nonadjacent dependencies by multiple-cue integration. Journal of Memory and Language. 2012 Nov 30;67(4):507-20.

40. Christiansen MH. Language has evolved to depend on multiple-cue integration. The evolutionary emergence of language: Evidence and Inference. 2013 Jul 25:42-61.

41. Endress AD, Dehaene-Lambertz G, Mehler J. Perceptual constraints and the learnability of simple grammars. Cognition. 2007 Dec 31;105(3):577-614.

42. Jones MN, Mewhort DJ. Representing word meaning and order information in a composite holographic lexicon. Psychological review. 2007 Jan;114(1):1.

43. Marcus GF, Vijayan S, Rao SB, Vishton PM. Rule learning by seven-month-old infants. Science. 1999 Jan 1;283(5398):77-80.

44. Saffran JR, Pollak SD, Seibel RL, Shkolnik A. Dog is a dog is a dog: Infant rule learning is not specific to language. Cognition. 2007 Dec 31;105(3):669-80.

45. Amato MS, MacDonald MC. Sentence processing in an artificial language: Learning and using combinatorial constraints. Cognition. 2010 Jul 31;116(1):143-8.

46. Goldberg AE. Constructions: a new theoretical approach to language. Trends in cognitive sciences. 2003 May 31;7(5):219-24.

47. Wonnacott E, Newport EL, Tanenhaus MK. Acquiring and processing verb argument structure: Distributional learning in a miniature language. Cognitive psychology. 2008 May 31;56(3):165-209.

48. Bower GH. Organizational factors in memory. Cognitive psychology. 1970 Jan 31;1(1):18-46.

49. McRae K, De Sa VR, Seidenberg MS. On the nature and scope of featural representations of word meaning. Journal of Experimental Psychology: General. 1997 Jun;126(2):99. 
50. Smith EE, Shoben EJ, Rips LJ. Structure and process in semantic memory: A featural model for semantic decisions. Psychological review. 1974 May;81(3):214.

51. Kemler-Nelson D, Jusczyk PW, Mandel DR, Myers J, Turk A, Gerken L. The head-turn preference procedure for testing auditory perception. Infant behavior and development. 1995 Jan 1;18(1):111-6.

52. Lakoff G. Women, fire, and dangerous things. University of Chicago press; 2008 Aug 8.

53. Collins AM, Loftus EF. A spreading-activation theory of semantic processing. Psychological review. 1975 Nov;82(6):407.

54. Deese J. On the structure of associative meaning. Psychological review. 1962 May;69(3):161.

55. Garnsey SM, Pearlmutter NJ, Myers E, Lotocky MA. The contributions of verb bias and plausibility to the comprehension of temporarily ambiguous sentences. Journal of Memory and Language. 1997 Jul 31;37(1):5893.

56. Gómez RL. Variability and detection of invariant structure. Psychological Science. 2002 Sep;13(5):431-6.

57. Landauer TK, Dumais ST. A solution to Plato's problem: The latent semantic analysis theory of acquisition, induction, and representation of knowledge. Psychological review. 1997 Apr;104(2):211.

58. Burgess $\mathrm{C}$, Lund $\mathrm{K}$. Modelling parsing constraints with high-dimensional context space. Language and cognitive processes. 1997 Mar 1;12(2-3):177-210.

59. Dale PS, Fenson L. Lexical development norms for young children. Behavior Research Methods, Instruments, \& Computers. 1996 Mar 1;28(1):125-7.

60. Arias-Trejo N, Plunkett K. Lexical-semantic priming effects during infancy. Philosophical Transactions of the Royal Society of London B: Biological Sciences. 2009 Dec 27;364(1536):3633-47.

61. Willits JA, Wojcik EH, Seidenberg MS, Saffran JR. Toddlers activate lexical semantic knowledge in the absence of visual referents: evidence from auditory priming. Infancy. 2013 Nov 1;18(6):1053-75.

62. Keppel, G., \& Wickens, TD. Design and Analysis: A Researcher's Handbook, 4th

63. Cohen LB, Atkinson DJ, Chaput HH. Habit 2000: A new program for testing infant perception and cognition (Version 2.2.5c)[Computer software] University of Texas.

64. Edition. 2004. Upper Saddle River, NJ : Prentice Hall.

65. Mandler G, Pearlstone Z, Koopmans HS. Effects of organization and semantic similarity on recall and recognition. Journal of Verbal Learning and Verbal Behavior. 1969 Jun 30;8(3):410-23.

66. Underwood BJ, Schulz RW. Meaningfulness and verbal learning. 1960.

67. Kramer JH, Delis DC, Kaplan E, O'Donnell L, Prifitera A. Developmental sex differences in verbal learning. Neuropsychology. 1997 Oct;11(4):577.

68. Lany J, Gómez RL. Prior experience shapes abstraction and generalization in language acquisition. In Generalization of Knowledge: Multidisciplinary Perspectives. 2011 Jan 1. Taylor and Francis.

69. Haskell TR, Thornton R, MacDonald MC. Experience and grammatical agreement: Statistical learning shapes number agreement production. Cognition. $2010 \mathrm{Feb} 28 ; 114(2): 151-64$.

70. Howard MW, Kahana MJ. When does semantic similarity help episodic retrieval?. Journal of Memory and Language. 2002 Jan 31;46(1):85-98.

71. Johnson SP, Fernandes KJ, Frank MC, Kirkham N, Marcus G, Rabagliati H, Slemmer JA. Abstract Rule Learning for Visual Sequences in 8-and 11-Month-Olds. Infancy. 2009 Jan 2;14(1):2-18.

72. Thiessen ED, Hill EA, Saffran JR. Infant-directed speech facilitates word segmentation. Infancy. 2005 Jan 1;7(1):53-71.

73. Thiessen ED, Saffran JR. When cues collide: use of stress and statistical cues to word boundaries by 7-to 9month-old infants. Developmental psychology. 2003 Jul;39(4):706.

74. Wojcik EH, Saffran JR. The ontogeny of lexical networks: Toddlers encode the relationships among referents when learning novel words. Psychological science. 2013 Oct;24(10):1898-905.

75. Ferretti TR, McRae K, Hatherell A. Integrating verbs, situation schemas, and thematic role concepts. Journal of Memory and Language. 2001 May 31;44(4):516-47.

76. Marchman VA, Fernald A, Hurtado N. How vocabulary size in two languages relates to efficiency in spoken word recognition by young Spanish-English bilinguals. Journal of Child Language. 2010 Sep;37(4):817-40.

77. Endress AD, Scholl BJ, Mehler J. The role of salience in the extraction of algebraic rules. Journal of Experimental Psychology: General. 2005 Aug;134(3):406.

78. Turk-Browne NB, Isola PJ, Scholl BJ, Treat TA. Multidimensional visual statistical learning. Journal of Experimental Psychology: Learning, Memory, and Cognition. 2008 Mar;34(2):399.

79. Fisher C, Gleitman H, Gleitman LR. On the semantic content of subcategorization frames. Cognitive psychology. 1991 Jul 31;23(3):331-92. 
80. Willits JA, Amato MS, MacDonald MC. Language knowledge and event knowledge in language use. Cognitive psychology. 2015 May 31;78:1-27.

81. Riordan B, Jones MN. Redundancy in perceptual and linguistic experience: Comparing feature-based and distributional models of semantic representation. Topics in Cognitive Science. 2011 Apr 1;3(2):303-45.

82. Fernald A, Marchman VA. Individual differences in lexical processing at 18 months predict vocabulary growth in typically developing and late-talking toddlers. Child development. 2012 Jan 1;83(1):203-22.

83. Cleeremans A, McClelland JL. Learning the structure of event sequences. Journal of Experimental Psychology: General. 1991 Sep;120(3):235.

84. Hare M, McRae K, Elman JL. Sense and structure: Meaning as a determinant of verb subcategorization preferences. Journal of Memory and Language. 2003 Feb 28;48(2):281-303.

85. Battig, WF. Paired-associate learning. 1972. General Learning Corp: Morristown, NJ.

86. Baddeley AD. The influence of acoustic and semantic similarity on long-term memory for word sequences. The Quarterly Journal of Experimental Psychology. 1966 Nov 1;18(4):302-9.

87. Cofer CN, Foley JP. Mediated generalization and the interpretation of verbal behavior: I. Prolegomena. Psychological Review. 1942;49(6):513-40.

88. Rice UM, Di Vesta FJ. A developmental study of semantic and phonetic generalization in paired-associate learning. Child Development. 1965 Sep 1:721-30.

89. Gulya M, Rovee-Collier C, Galluccio L, Wilk A. Memory processing of a serial list by young infants. Psychological Science. 1998 Jul;9(4):303-7.

90. Huebner PA, Willits, JA. Structured semantic knowledge can emerge automatically from predicting word sequences in child-directed speech. Manuscript under review. DOI:10.17605/OSF.IO/QHWV5. https://psyarxiv.com/qhwv5/.

91. Nelson DL, McKinney VM, Gee NR, Janczura GA. Interpreting the influence of implicitly activated memories on recall and recognition. Psychological review. 1998 Apr;105(2):299.

92. Tulving E. Subjective organization in free recall of "unrelated" words. Psychological review. 1962 Jul;69(4):344.

93. Anderson JR, Bothell D, Lebiere C, Matessa M. An integrated theory of list memory. Journal of Memory and Language. 1998 May 31;38(4):341-80.

94. Raaijmakers JG, Shiffrin RM. Search of associative memory. Psychological review. 1981 Mar;88(2):93.

95. Ratcliff R, McKoon G. A retrieval theory of priming in memory. Psychological review. 1988 Jul;95(3):385.

96. Saffran JR, Aslin RN, Newport EL. Statistical learning by 8-month-old infants. Science. 1996 Dec 13:1926-8.

97. Saffran JR. The use of predictive dependencies in language learning. Journal of Memory and Language. 2001 May 31;44(4):493-515.

98. Wells JB, Christiansen MH, Race DS, Acheson DJ, MacDonald MC. Experience and sentence processing: Statistical learning and relative clause comprehension. Cognitive psychology. 2009 Mar 31;58(2):250-71.

99. Misyak JB, Christiansen MH, Tomblin JB. On-line individual differences in statistical learning predict language processing. Frontiers in psychology. 2010;1.

100.Katz JJ, Fodor JA. The structure of a semantic theory. language. 1963 Apr 1;39(2):170-210.

101.Hunter MA, Ames EW. A multifactor model of infant preferences for novel and familiar stimuli. Advances in infancy research. 1988.

102.Lany J, Saffran JR. From statistics to meaning: Infants' acquisition of lexical categories. Psychological Science. $2010 \mathrm{Feb} ; 21(2): 284-91$. 


\author{
Experiment 1 \\ Semantic \\ Familiarization \\ cookie-towel-banana \\ cookie-soap-banana \\ cookie-toothbrush-banana \\ dog-towel-kitty \\ dog-soap-kitty \\ dog-toothbrush-kitty

\section{Match}

Control Familiarization

cookie-towel-kitty

cookie-soap-kitty

cookie-toothbrush-kitty

dog-towel-banana

dog-soap-banana

dog-toothbrush-banana

\section{Experiment 2 \\ Semantic \\ Familiarization \\ cookie-shoe-banana \\ cookie-keys-banana \\ cookie-phone-banana \\ dog-shoe-kitty \\ dog-keys-kitty \\ dog-phone-kitty}

Experiment 3

Semantic

Familiarization

toast-towel-cheese

toast-soap-cheese

toast-toothbrush-cheese

birdy-towel-fish

birdy-soap-fish

birdy-toothbrush-fish

Experiment 4

Semantic

Familiarization

toast-shoe-banana

toast-keys-banana

toast-phone-banana

birdy-shoe-fish

birdy-keys-fish

birdy-phone-fish
Match Control Familiarization

cookie-shoe-kitty

cookie-keys-kitty

cookie-phone-kitty

dog-shoe-banana

dog-keys-banana

dog-phone-banana

Match

Control Familiarization

toast-towel-fish

toast-soap-fish

toast-toothbrush-fish

birdy-towel-cheese

birdy-soap-cheese

birdy-toothbrush-cheese

Match

\section{Control Familiarization}

toast-shoe-fish

toast-keys-fish

toast-phone-fish

birdy-shoe-cheese

birdy-keys-cheese

birdy-phone-cheese

\section{Test Stimuli}

cookie-soap-banana

cookie-toothbrush-banana

dog-soap-kitty

dog-toothbrush-kitty

cookie-soap-kitty

cookie-toothbrush-kitty

dog-soap-banana

dog-toothbrush-banana

\section{Test Stimuli}

Same as Experiment 1

\section{Test Stimuli}

Same as Experiment 1

\section{Test Stimuli}

Same as Experiment 1 